\title{
FEASIBILITY OF CLINICAL TRIAL RECRUITMENT FOR CEREBRAL AMYLOID ANGIOPATHY: A SPECIALIST SINGLE CENTRE EXPERIENCE
}

Gargi Banerjee ${ }^{\mathrm{a}}$, David J Werringa .

aStroke Research Centre, Department of Brain Repair and Rehabilitation, UCL Queen Square Institute of Neurology and the National Hospital for Neurology and Neurosurgery, London, UK

\section{Corresponding author:}

Professor David J Werring

Stroke Research Centre, Department of Brain Repair and Rehabilitation, UCL Queen

Square Institute of Neurology, Russell Square House, 10 - 12 Russell Square, London WC1B 5EH, UK.

Tel: +44 (0)20 3108 7493; Fax: +44 (0)20 7833 8613; Email: d.werring@ucl.ac.uk

Manuscript length: 1607 words

\section{Keywords:}

Cerebral amyloid angiopathy; trial recruitment. 


\begin{abstract}
Introduction:

Cerebral amyloid angiopathy (CAA) is a small vessel disease characterised by vascular amyloid-beta deposition and recurrent intracerebral haemorrhage, for which there are limited data on the practicalities of clinical trial recruitment.
\end{abstract}

\title{
Methods:
}

We describe our single centre recruitment experience for a small biomarker pilot study, which aimed to recruit 10 patients with CAA.

\section{Results:}

The BOCAA study recruited 10 CAA patients over 18 months. All patients were recruited from a prospective CAA database $(n=186)$; the majority of patients $(n=146$, $78.5 \%$ ) were ineligible for the BOCAA study. The most common reasons for exclusion were co-existent cognitive impairment or dementia $(n=42)$, failure to meet the imaging (modified Boston) criteria $(n=41)$, and anticoagulant or dual antiplatelet use $(n=18)$.

\section{Conclusions:}

Recruitment of CAA patients to a small pilot study is feasible from a single specialist centre; however, centralised multicentre research databases will allow for more effective and co-ordinated recruitment to larger studies. Any future trial will need to consider how best to define mild disease, factors that influence group heterogeneity, and the impact of comorbidities that could limit participation in multimodal testing but be mindful that more stringent entry criteria will limit recruitment capabilities. 


\section{INTRODUCTION}

A number of high profile trials in Alzheimer's disease have failed to demonstrate disease-modifying effects [1, 2]; these failures have led to a critical appraisal of how these trials are conducted, and have highlighted the importance of selecting appropriate patients for study enrolment (ideally those with early or mild disease, where potential for disease modification remains possible), and the need for reliable, clinically meaningful, outcome markers [1, 2]. Cerebral amyloid angiopathy (CAA), a cerebral small vessel disease characterised by vascular amyloid-beta deposition and recurrent intracerebral haemorrhage (ICH) [3], not only shares a pathological protein with Alzheimer's disease but also similar challenges in designing effective trials. Whilst the need for accurate outcome markers is recognised [4], less has been said about identifying appropriate patients with CAA for trial recruitment [5].

We describe our single centre experience of recruiting CAA patients to a small biomarker pilot study. We wished to establish whether it was feasible to identify patients with mild disease, who were able to tolerate the multimodal testing specified by the study protocol.

\section{MATERIAL \& METHODS}

The BOCAA (Biomarkers and Outcomes in Cerebral Amyloid Angiopathy) study was conducted at the National Hospital for Neurology and Neurosurgery (NHNN), Queen Square, London. Ethical approval was granted in October 2015 (REC reference 15/LO/1443). Written consent was obtained for all participants. The study aimed to recruit 10 patients with CAA and 5 age matched healthy controls. 
The study required three visits. The first was a screening visit, where the eligibility criteria were reviewed and the participant was consented. The second visit included a clinical assessment (full history, neurological examination, baseline observations, Montreal Cognitive Assessment), blood tests, formal neuropsychological testing and PET-MR imaging (using the amyloid ligand ${ }^{18} \mathrm{~F}$-florbetapir, "Amyvid"). On the third visit, the participant would have a lumbar puncture (LP).

For CAA patients, the inclusion criteria were: age $\geq 55$ years, Mini-Mental State Examination score $\geq 23$, modified Rankin scale $(m R S) \leq 3$ and capacity to give informed consent. Patients with CAA were required to meet "probable" modified Boston Criteria [6]. Those with absolute or relative contraindications to PET or MRI scanning or lumbar puncture (including anticoagulant or dual antiplatelet use) were excluded. Additionally, we did not include patients with intracranial implants or other devices that could cause MR artefact, or patients with significant visual impairment (as the imaging protocol included visual task functional MRI).

Two methods were used to identify potentially eligible CAA patients. The first involved screening a prospectively collected database of CAA patients (listed from July 2008 to present). This database was part of a Research Ethics Committee approved Service Evaluation Agreement, which provides permission to use anonymised data for patients with intracranial haemorrhage known to the clinical stroke service. The database primarily includes those attending specialist intracerebral haemorrhage clinics at NHNN or University College Hospital $(\mathrm{UCH})$, London. It also includes private patients and patients from other centres who are 
interested in participating in research. The vast majority of patients in this database were outpatients with CAA (95\%). The second method was screening patients presenting with lobar intracerebral haemorrhage to the Hyperacute Stroke Unit (HASU), UCH, and Acute Brain Injury Unit (ABIU), NHNN.

The steps after identification of a potentially eligible patient are shown in Figure 1.

\section{RESULTS}

The BOCAA study reached its recruitment target of 10 CAA patients within 18 months. All patients were recruited from the prospective CAA database; no patients were directly recruited from the HASU or ABIU (inpatient units), although two patients who were admitted to the HASU and ABIU and later seen by the outpatient service were contacted. Six months after recruitment opened, direct recruitment from the HASU and ABIU was abandoned.

The reasons for patient exclusions are shown in Figure 2. A large proportion $(n=146$, $78.5 \%$ ) of patients within our CAA database were ineligible for the BOCAA study. Most patients were excluded following electronic case note review, with the commonest reason being cognitive impairment or dementia $(n=42)$. Other common reasons for exclusion were failure to meet the imaging criteria $(n=41)$, and anticoagulant or dual antiplatelet use $(n=18)$. Of the 40 patients invited to attend the screening appointment, 20 patients responded; of these, 10 patients declined to participate, and 10 were successfully recruited. 


\section{DISCUSSION}

We believe that our experience of CAA patient recruitment raises important points for consideration. Firstly, we reached our recruitment target (10 CAA patients, over a period of 18 months), demonstrating that this level of recruitment is feasible for a single specialist centre. Secondly, we found that recruiting patients acutely was difficult (no patients were recruited directly from our inpatient unit), and that recruitment using a prospective outpatient research database was more effective. Finally, we showed that a large proportion ( $n=146,78.5 \%)$ of patients within our CAA database were ineligible for the BOCAA study. Given that data on recruiting patients with CAA for clinical trials is limited [5], this work makes an important contribution by highlighting a number of practical aspects which may inform future larger trials.

In our experience, there were two barriers to recruiting CAA patients after acute presentation with $\mathrm{ICH}$ (i.e. those admitted to the HASU or ABIU). Firstly, patients were often critically unwell, with the likelihood of functional recovery uncertain. It is also likely that early post-ICH mortality contributed to a lack of recruitment from these inpatient units. Secondly, many patients were discharged from the hyperacute unit prior to having an MRI. These discharged patients were likely to have an MRI when followed up by their local stroke unit; some of them would no longer be under the care of the $\mathrm{NHNN/UCH}$ service, and therefore our centre would not have routine access to further clinical details or imaging data. This is a limitation of the "hub and spoke" model for acute stroke care [7], and demonstrates the constraints of MR based diagnostic criteria. A centralised multicentre research database, compiled from standardised local databases, is likely to allow more effective and co-ordinated 
recruitment to future CAA studies. Additionally, the introduction of CT-based diagnostic criteria [8] might improve the ability to identify CAA patients in the acute setting, although the diagnostic accuracy of these criteria in people who survive intracerebral haemorrhage has not yet been investigated.

Our recruitment process took longer than originally projected; we had initially estimated that full recruitment would take between 6 and 12 months. To give an indication of our specialist ICH clinic attendance, there were a total of 1192 clinic visits made by 663 individual patients between January $1^{\text {st }} 2015$ and June $1^{\text {st }} 2018$. We believe that our recruitment delay was due to the stringency of our study inclusion and exclusion criteria, which were designed to identify patients with clinically mild CAA (there are currently no consensus criteria for CAA clinical severity) and to reduce group heterogeneity. Over a quarter of clinically eligible patients were excluded following imaging review, with most not fully meeting the modified Boston Criteria or demonstrating evidence of co-existent deep perforator ("hypertensive") arteriopathy (arteriolosclerosis). Patients were similarly excluded if their electronic case records noted "mixed" small vessel disease; we also excluded those with Alzheimer's disease, which again frequently occurs with CAA [3]. This highlights the difficulties of identifying CAA in isolation, a key point for future trials. We also excluded a number of patients who would be unable to fully participate in the study protocol, for example due to anticoagulant or dual anti-platelet use (as a relative contraindication for LP), significant visual impairment, absolute MRI contraindications, and high mRS scores. The existence of comorbidities that either complicate the diagnosis of CAA or limit patient participation in a multimodal protocol will have relevance for larger clinical studies. 
We found that, overall, co-existent cognitive impairment or dementia was the most common reason for exclusion. We included this in the entry criteria as a surrogate marker for mild disease, and to ensure that patients were able to provide consent, in particular for the LP, and to comply with functional MRI studies. Interventional studies are likely to require repeated visits and obtaining consent for invasive procedures during the course of a trial might be challenging in patients with significant cognitive difficulties at study entry. If future studies relax these criteria, they will need to ensure arrangements for continuing participation if a patient loses capacity to provide consent after study entry.

We acknowledge that there are aspects of our work which limit its generalisability. Our experience with cerebral amyloid angiopathy is not necessarily generalisable to other cerebral small vessel diseases. Additionally, it is likely that some of the recruitment limitations we encountered are unique to our (single) centre, and would not be experienced elsewhere, particularly those with a simpler or more integrated follow up pathway after discharge. Other centres might instead be susceptible to enrolment barriers that we did not encounter or define. Given that we were using an historical database, we were necessarily obliged to recruit participants by letter or telephone; this might be less effective than direct recruitment by a physician or research practitioner in clinic, where questions and concerns can be addressed directly.

\section{CONCLUSIONS}


We conclude that recruitment of CAA patients to a small pilot study is feasible from a single specialist centre; however, centralised multicentre research databases will allow for more effective and co-ordinated recruitment to larger studies. Any future trial will need to consider how best to define mild disease, factors that influence group heterogeneity, and the impact of comorbidities that could limit participation in multimodal testing - but be mindful that more stringent entry criteria will limit recruitment. Further data from other centres on their experiences of recruiting for CAA studies are needed to determine the generalisability of our findings.

\section{ACKNOWLEDGEMENTS}

The BOCAA study was funded by the Rosetrees Trust and the Leonard Wolfson Experimental Neurology Centre (LWENC) Biomarker Research Group. GB holds an NIHR Academic Clinical Fellowship and received funding from the Rosetrees Trust. DJW receives research support from the Stroke Association, the British Heart Foundation and the Rosetrees Trust. We would like to acknowledge clinical support from the LWENC Clinical Research Facility at NHNN. This work was undertaken at UCLH/UCL which receives a proportion of funding from the Department of Health's National Institute for Health Research (NIHR) Biomedical Research Centres funding scheme.

\section{COMPETING INTERESTS}

The authors report no disclosures or conflicts of interest relevant to the manuscript. 


\section{REFERENCES}

[1] T.E. Golde, S.T. DeKosky, D. Galasko, Alzheimer's disease: The right drug, the right time, Science (New York, N.Y.) 362(6420) (2018) 1250-1251.

[2] E. Alteri, L. Guizzaro, Be open about drug failures to speed up research, Nature 563(7731) (2018) 317-319.

[3] G. Banerjee, R. Carare, C. Cordonnier, S.M. Greenberg, J.A. Schneider, E.E. Smith, M.V. Buchem, J.V. Grond, M.M. Verbeek, D.J. Werring, The increasing impact of cerebral amyloid angiopathy: essential new insights for clinical practice, Journal of neurology, neurosurgery, and psychiatry 88(11) (2017) 982-994.

[4] S.M. Greenberg, R. Al-Shahi Salman, G.J. Biessels, M. van Buchem, C. Cordonnier, J.M. Lee, J. Montaner, J.A. Schneider, E.E. Smith, M. Vernooij, D.J. Werring, Outcome markers for clinical trials in cerebral amyloid angiopathy, The Lancet. Neurology 13(4) (2014) 419-28.

[5] C. Leurent, J.A. Goodman, Y. Zhang, P. He, J.R. Polimeni, M.E. Gurol, M. Lindsay, L. Frattura, U.S. Sohur, A. Viswanathan, M.M. Bednar, E.E. Smith, G. Ponezumab Trial Study, S.M. Greenberg, Immunotherapy with ponezumab for probable cerebral amyloid angiopathy, Annals of clinical and translational neurology 6(4) (2019) 795-806.

[6] J. Linn, A. Halpin, P. Demaerel, J. Ruhland, A.D. Giese, M. Dichgans, M.A. van Buchem, H. Bruckmann, S.M. Greenberg, Prevalence of superficial siderosis in patients with cerebral amyloid angiopathy, Neurology 74(17) (2010) 1346-50. [7] J.K. Elrod, J.L. Fortenberry, Jr., The hub-and-spoke organization design: an avenue for serving patients well, BMC Health Serv. Res. 17(Suppl 1) (2017) 457. 
[8] M.A. Rodrigues, N. Samarasekera, C. Lerpiniere, C. Humphreys, M.O. McCarron, P.M. White, J.A.R. Nicoll, C.L.M. Sudlow, C. Cordonnier, J.M. Wardlaw, C. Smith, R. Al-Shahi Salman, The Edinburgh CT and genetic diagnostic criteria for lobar intracerebral haemorrhage associated with cerebral amyloid angiopathy: model development and diagnostic test accuracy study, The Lancet. Neurology 17(3) (2018) 232-240. 


\section{FIGURES}

Figure 1:

Flowchart detailing the process between identification of a potentially eligible patient and their recruitment

\section{Figure 2:}

Reasons for ineligibility

\section{Abbreviations:}

AD, Alzheimer's disease; CAA, cerebral amyloid angiopathy; cSS, cortical superficial siderosis; DPA, deep perforator arteriopathy; ICH, intracerebral haemorrhage; LP, lumbar puncture; MR, magnetic resonance (imaging); mRS, modified Rankin Scale; SVD, small vessel disease. 